\title{
The relationship between attention-deficit/ hyperactivity disorder and trauma in adolescents
}

\author{
Ali Reza Shafiee-Kandjani ${ }^{1}$, Seyed Gholamreza Noorazar ${ }^{2 *}$, Saeed Aslanabadi ${ }^{3}$, Nasim Rashedi ${ }^{2}$, Mehrnaz \\ Dadkhah², Mohsen Jafarzadeh-Gharehziaaddin ${ }^{4}$
}

${ }^{1}$ Road Traffic Injury Research Center (RTIR), Tabriz University of Medical Sciences, Tabriz, Iran

${ }^{2}$ Research Center of Psychiatry and Behavioral Sciences, Tabriz University of Medical Sciences, Tabriz, Iran

${ }^{3}$ Department of Surgery, Tabriz University of Medical Sciences, Tabriz, Iran

${ }^{4}$ Department of Psychology, Islamic Azad University of Ahar, Tabriz, Iran

\author{
Received: 18 April 2017 \\ Accepted: 25 June 2017 \\ Published online: 1 July 2017 \\ *Corresponding author: Seyed \\ Gholamreza Noorazar, Department \\ of Psychiatry, Razi Mental Hospital, \\ El Goli Boulevard, PO Box: 5456, \\ Tabriz, Iran. \\ Tel/Fax: +98 4133803351 \\ Email:s.gh.noorazar@gmail.com \\ Competing interests: None. \\ Funding information: This paper \\ has been extracted from the MD \\ dissertation written by Mrs. Nassim \\ Rashedi and has been funded by \\ the Research Center of Psychiatry \\ and Behavioral Sciences affiliated \\ to Tabriz University of Medical \\ Sciences. \\ Citation: Shafiee-Kandjani AR, \\ Noorazar SG, Aslanabadi S, Rashedi \\ N, Dadkhah M, Jafarzadeh- \\ Gharehziaaddin M. The relationship \\ between attention-deficit/ \\ hyperactivity disorder and trauma in \\ adolescents. Journal of Emergency \\ Practice and Trauma 2018; 4(1): 18 \\ 23. doi: 10.15171/jept.2017.23.
}

\begin{abstract}
Objective: Attention-deficit/hyperactivity disorder (ADHD) is characterized by persistent and impairing levels of inattention, impulsivity and hyperactivity. Evidence shows that adolescents with ADHD are more exposed to trauma. This study aimed to investigate the relationship between ADHD symptom severity and trauma severity.

Methods: In this descriptive correlational study, the study population included traumatic adolescents aged 12-18 years referred to Shohada hospital in Tabriz, Iran in 2016. Among this population, 91 subjects were selected. In order to exclude subjects with other psychiatric disorders, a psychiatrist performed clinical interviews with them. In this regard, the short form of Conner's Comprehensive Behavior Rating Scales Revised Edition (CBRS-R) and Pediatric Trauma Scale were used. For data analysis, Pearson correlation coefficient and independent $t$ test were applied. Data were analyzed using SPSS software version 22 . Results: There was a significant positive relationship between trauma severity and ADHD score, hyperactivity, and oppositional/Impulsivity $(P<0.01)$. Conversely, no statistical significance was observed between attention deficiency and trauma severity. The severity of trauma was higher among ADHD group than normal individuals. There were also correlations between socioeconomic status (SES) and oppositional/impulsive patients. In this regard, higher scores of oppositional/impulsivity were observed among patients with lower SES.

Conclusion: Traumas have significant effects on economic and humanistic aspects of life in modern era. Our findings showed that there was a statistically significant relationship between hyperactivity-inattention and trauma intensity in adolescents. Therefore, to prevent traumatic events, ADHD screening at schools is suggested. By the same token, informing parents through mass media can help reduce the consequences of inattention/ hyperactivity disorder in the society.

Keywords: Hyperactivity, Trauma severity, Attention deficit, Impulsivity, ADHD
\end{abstract}

\section{Introduction}

Attention-deficit/hyperactivity disorder (ADHD) is one of the most common disorders which happens mostly at school years with symptoms like inattentiveness, hyperactivity, impulsive behaviors, or a combination of these symptoms (1). It may impair cognitive abilities and psycho-social functions. Not only it can have some effects on children and adolescents' life, but also it can affect the family's quality of life (2).The Diagnostic and Statistical Manual of Mental Disorders Fifth Edition (DSM-5) specifies this disorder based on two aspects of symptoms: 1) the aspect of attention deficiency and 2) hyperactivity and impulsiveness. Thus, it is necessary to control these symptoms in adolescents as they can cause some problems at school or in social places for them. Also, DSM5 introduces three subcategories (presentations) for ADHD: (a) inattentive, $(b)$ hyperactive/impulsive and (c) combined (3). ADHD children may experience significant problems in adaptability because of their expected level of development or growth with their chronological age (4). In $60 \%$ of cases, symptoms rests until the adulthood stage and most cases are concomitant with other mental disorders such as depression, anxiety, obsession, learning disabilities, bipolar disorder, and so on (5-7). In some studies, the education failure and background emotional problems such as anxiety and depression were reported 
in adolescents with ADHD (8-11). Evidence shows that children and adolescents with ADHD are more exposed to traumas. Therefore, without doing therapeutic interventions, this disorder could result in car accidents and head traumas $(12,13)$. Other studies revealed that this disorder increases the danger of accidents and injuries to pedestrian adolescents. Weak attention skills and high impulsiveness, while crossing streets, could be the reasons underlying such injuries (14). Many studies have been done throughout the world about children and adolescents with ADHD and trauma. In England, about 18000 people lose their life yearly due to accidents and more than half of them are adolescents and young people under 30 (15). Such studies indicate that most of traumatic cases are males, adolescents and young people. The basic causes of traumatic diseases for younger people, especially in developing countries, are workhouse accidents resulted from low security of those places and road accidents.

Currently, about $50 \%$ of trauma cases throughout the world are car and traffic accidents. Also, according to World Health Organization (WHO), inattention to this significant problem will increase this number to 60 by 2020. Three main causes of mortality in the world are cardiovascular diseases, cancer and trauma. Trauma is the second cause of fatality in Iran (16). Impulsivity, inattentiveness and executive dysfunctions are factors which lead ADHD adolescents toward dangerous behaviors and that may result in injuries (17). In addition, studies show that people with ADHD in comparison with their peers have more tendencies for doing dangerous behaviors (18). As there is scant evidence in this area and due to the priority of Iranian health system in preventing morbidity and mortality because of injuries and road accidents, we decided to conduct a study to investigate the relationship between hyperactivity/inattention and trauma among adolescents referred to Shohada hospital in Tabriz, Iran. We aimed to know whether ADHD symptoms were correlated with the severity of trauma in adolescents.

\section{Methods}

This was a descriptive correlational study. Participants included traumatic adolescents aged 12-18 years who referred to Shohada hospital in Tabriz in 2016. In order to determine the sample size, we conducted a Pilot study with 30 samples. After measuring the correlation between variables, the final sample size of 91 was obtained at $\alpha=0.05$, power of $80 \%$. Subjects were selected using convenience sampling technique. The inclusion criteria encompassed patients aged 6-12 years who were hospitalized for trauma due to falling, cycling, riding motorbike, and pedestrian accidents. The exclusion criteria were unwillingness to participate, major psychiatric disorders (e.g. psychosis) at the time of accident, medical disorders leading to clouding of consciousness at the time of accidents (e.g. seizures), indirect role of an adolescent in the occurrence of traumas (e.g. vehicle accidents or fights caused by other people), IQ less than 75 (Raven's Colored Progressive Matrices
Test), consumption history of antiepileptic drugs, and medications with an impact on the brain. For rejecting other psychiatric disorders, the subjects were clinically interviewed.

This questionnaire has 27 items with four subscales which is completed by parents. Oppositional, cognitive problems/inattentiveness, hyperactivity and ADHD index .Standard score equals or more than 59 shows significant clinical problems in that subscale. Coefficient internal reliability with a range of 0.75 to 0.90 were reported. The validity of structures in Conner's form was obtained by factor analysis and its differential validity was obtained by a survey on statistical capability of the questionnaire in discriminating people with ADHD from normal individuals and other clinical groups (19).

Evidence indicates that Pediatric Trauma Scale (PTS) is a valid tool for predicting traumatic injuries which result in death. The score above 8 in this questionnaire reveals the probability of $9 \%$ death and scores below or equal to zero clarifies the probability of $100 \%$ death. Between low scores in PTS and the death probability, there is a linear relationship. The lowest score of questionnaire is 6 and the highest is 12. Trauma Severity Score of 7-11 shows minor or slight injuries, 1-6 shows moderate injuries and -6 shows severe and serious injuries (20).

For data analysis, Pearson correlation coefficient and independent $t$ test using SPSS software version 20 were utilized.

\section{Results}

From 91 people who participated in this study 13 were females ( 8 in ADHD group and 5 in normal group) and 78 were males (35 in ADHD group and 43 in normal group). The average age of participants was $12.90 \pm 2.22$ years (13.14 for normal group and $12.62 \pm 2.29$ for ADHD group). The average age for parents was 41.09 \pm 5.8 . Concerning parents' education, 57 (62.6\%) had guidance school certificate, 11 (12.1\%) had diploma, 10 (11\%) had associate degree, and 13 (14.3\%) had a license or higher degrees. Regarding parents' occupation, 53 (58.2\%) were employed, 11 (12.1\%) were unemployed and 27 (29.7\%) were housewives. In terms of income, 53 (58.2\%) had less than $\$ 255$ monthly and 38 (41.8\%) had more than $\$ 255$ per month. With regard to housing, 34 (37.4\%) parents had a renting house and $57(62.6 \%)$ parents owned a house. Socioeconomic status (SES) of 46 participants was weak (15 people in ADHD and 31 in normal group), 31 participants were about average (16 people in ADHD and 15 people in normal group) and 14 participants were in good economical and social conditions (14 people in ADHD and 2 in normal group) (Table 1).

In Table 2, average, standard deviation, the highest and lowest variable scores are represented in two different ADHD and normal groups.

As can be seen from Table 3, 36 adolescents had lacerations (16 in ADHD group and 20 in normal group), 41 had closed fractures (22 in ADHD group and 19 in normal group), and 14 had open fractures (4 in ADHD group and 
Table 1. Demographic information of participants and their parents in this study

\begin{tabular}{|c|c|c|c|}
\hline & ADHD group & Normal group & All \\
\hline \multicolumn{4}{|l|}{ Adolescents sex } \\
\hline Female, No. \% & $8(18.6)$ & $5(10.4)$ & $13(14.3)$ \\
\hline Male, No. \% & $35(81.4)$ & $43(89.6)$ & $78(85.7)$ \\
\hline $\begin{array}{l}\text { Average age of } \\
\text { adolescents }\end{array}$ & $12.62 \pm 2.29$ & $13.14 \pm 2.15$ & $12.90 \pm 2.22$ \\
\hline \multicolumn{4}{|l|}{ Parents sex } \\
\hline Female, No. $\%$ & $22(51.2)$ & $14(29.2)$ & $36(39.6)$ \\
\hline Male, No. \% & $21(48.8)$ & $34(70.8)$ & $55(60.4)$ \\
\hline $\begin{array}{l}\text { Average age of } \\
\text { parents }\end{array}$ & $40.44 \pm 6.23$ & $41.67 \pm 5.45$ & $41.09 \pm 5.8$ \\
\hline
\end{tabular}

10 in normal group). Adolescents in this study suffered from mild traumas.

Tables 4 and 5 show the frequency and severity of ADHD subtypes as well as types of traumas in ADHD patients in comparison with non-ADHD adolescents.

There was a significant negative correlation between ADHD Oppositional/impulsive components with social and economic situations (Table 6). Correlation matrix among trauma severity variables with ADHD score, hyperactivity, oppositional/impulsive, attention deficit brought results of oppositional/impulsive, hyperactivity and $\mathrm{ADHD}$ as follows $0.50,0.62$ and 0.61 respectively. According to the results, there was a positive significant relationship between trauma severity with ADHD score, hyperactivity, and oppositional/impulsive $(P<0.01)$. This indicates that with an increase in these variables, trauma severity increases as well. But there was no significant relationship between attention deficit and trauma severity $(P>0.05)$.

Independent $t$ test was used for calculating trauma severity in adolescents with ADHD and non-ADHD. Levene test for evaluating the equality of variances showed for variables and trauma severity: $F$, sig, $t, d f$ and again sig; $5.15,0.12,4.98,89,0.001$ respectively

The results of independent $t$ test showed that the meaningful differences therefore trauma severity in adolescents with ADHD and non-ADHD is different $(P=0.001, d f=89, t=4.98)$ and according to the Table 2 trauma severity in ADHD group is more than normal subjects.

\section{Discussion}

One of the most common behavioral disorders during childhood and adolescence is ADHD. This disorder involves approximately $3 \%$ to $5 \%$ of children and adolescents in school years. In this study, the relationship

Table 2. Descriptive index of variables studied in differentiated groups

\begin{tabular}{|c|c|c|c|c|c|c|}
\hline & Indexes & Average & Standard deviation & The lowest & The highest & Number \\
\hline \multirow{5}{*}{ ADHD group } & ADHD & 58.2 & 9.05 & 38 & 76 & 43 \\
\hline & Hyperactivity & 54 & 13.5 & 33 & 83 & 43 \\
\hline & Attention deficit & 54 & 13.19 & 37 & 87 & 43 \\
\hline & Oppositional/ impulsive & 54 & 8.5 & 40 & 77 & 43 \\
\hline & Trauma severity & 10.58 & 0.88 & 8 & 11 & 43 \\
\hline \multirow{5}{*}{ Normal group } & ADHD & 44.25 & 6.62 & 32 & 57 & 48 \\
\hline & Hyperactivity & 41.47 & 5.12 & 33 & 53 & 48 \\
\hline & Attention deficit & 44.33 & 7.88 & 33 & 57 & 48 \\
\hline & Oppositional/ impulsivity & 40.04 & 5.91 & 33 & 59 & 48 \\
\hline & Trauma severity & 9.89 & 0.57 & 9 & 11 & 48 \\
\hline
\end{tabular}

Table 3. Types of trauma frequency in different groups

\begin{tabular}{lccc}
\hline Types of trauma & ADHD group & Normal group & All \\
\hline Laceration & $16(38.1 \%)$ & $20(40.8 \%)$ & 36 \\
Closed Fracture & $22(52.4 \%)$ & $19(38.8 \%)$ & 41 \\
Open Fracture & $4(9.5 \%)$ & $10(20.4 \%)$ & 14 \\
\hline
\end{tabular}

Table 4. ADHD subtypes and severity in adolescents with ADHD

\begin{tabular}{lccc}
\hline ADHD subtypes & No. $\%$ & ADHD Severity & No. \% \\
\hline Inattentive Type & $12(28.6 \%)$ & Mild & $32(76.2 \%)$ \\
Hyperactive/impulsive type & $9(21.4 \%)$ & Severe & $8(19 \%)$ \\
Combined type & $21(50 \%)$ & Very severe & $2(4.8 \%)$ \\
\hline
\end{tabular}

Table 5. Trauma frequency in three types of ADHD

\begin{tabular}{lccc}
\hline Trauma type & Inattentive type & Hyperactivity/impulsivity type & Combined type \\
\hline Laceration, No. (\%) & $2(22.2)$ & $3(25)$ & $10(47.6)$ \\
Closed fracture, No. (\%) & $7(77.8)$ & $6(50)$ & $9(42.9)$ \\
Open fracture, No. (\%) & - & $3(25)$ & $2(9.5)$ \\
\hline
\end{tabular}


Table 6. Correlation matrix between trauma severity, ADHD, attention deficit, hyperactivity, and oppositional/impulsive components with economical and social situations

\begin{tabular}{llllll}
\hline & ADHD & Attention deficit & Hyperactivity & Oppositional/impulsive & Trauma severity \\
\hline Socio economic status & -0.38 & 0.08 & -0.13 & $-0.25^{*}$ & -0.16 \\
\hline
\end{tabular}

**Correlation is significant at the 0.01 level (2-tailed).

* Correlation is significant at the 0.05 level (2-tailed).

between the severity of hyperactivity/inattention symptoms and the severity of trauma in adolescents was investigated. The research findings showed that from 91 adolescents participated in the study, 13 were females and 78 were males. This ratio is in parallel with studies of Amiri et al (21), Abolhassanzadeh et al (22), Bener et al (23), and Mugnaini et al (24) as well as other studies on the prevalence of ADHD on higher incidence of this disorder in males than females. Evidence shows that the prevalence of inattention/hyperactivity disorder in males is higher than females. It is important to mention that the diagnosis of hyperactivity in males is faster and easier than females. This is due to the fact that the symptoms of ADHD are more significant in males and also males show more impulsive behavior compared with females. Thus, this disorder can be diagnosed faster in males. Generally, inattentiveness and ignorance of the surrounding issues are among the symptoms of this disorder in females with ADHD, which instead, impulsive behaviors are expected in males (25). Our findings revealed that 46, 31, and 14 people participated in this study were in poor, medium, and good economic and social status respectively. This is consistent with the findings by Esfandabadi et al (26), in which ADHD was most frequent in children with illiterate parents or with primary education. On the other hand, the least frequent rate was seen in parents with intermediate education or diploma. Parents' higher education contributed to the low frequency of ADHD.

Among 42 hyperactive adolescents, 50\% had combined $\mathrm{ADHD}, 28.6 \%$ suffered from inattentive hyperactivity, and $21.4 \%$ suffered from impulsive hyperactivity. This finding is consistent with the result obtained by Alishahi et al (27), Shahim et al (28). Conversely, it is inconsistent with the result by Abolhassanzadeh et al (22), in which they found the impulsive/hyperactive subtype as the most frequent in ADHD. This finding is mostly related to environmental, social, and cultural factors. Usually, the parents of hyperactive children are sensitive to the symptoms of attention deficit compared with impulsivity symptoms. Therefore, they refer to treatment centers following the incidence of these symptoms. In this regard, combined and inattentive types are more frequent than impulsive type in clinical settings. Another finding of the study suggested that the severity of the injury in all participants was mild. In addition, the results showed that there was a positive significant correlation between trauma severity and ADHD score, hyperactivity and opposition/impulsive scores $(P<0.01)$. This is in line with the findings by Schwebel et al, (17), Kaya et al (29), and Kouchakzadeh et al (30) which showed a meaningful association between
ADHD symptoms and the possibility of an increased risk of trauma.

The patients with adolescent and adult hyperactivity, are more exposed to injuries caused by vehicle crashes, so adults with serious traumas should be evaluated by a psychiatrist for being an ADHD.

However, in the present study no statistically significant relationship was found between inattention score and trauma severity, because the characteristics of hyperactive ADHD are running impulsivity in an inappropriate place, climbing up the wall and the restlessness (4). We can infer that such symptoms are highly correlated with the probability of injury and trauma in children and adolescents, while the characteristic of inattentive ADHD is associated with cognitive deficit rather than trauma or an injury, possibly due to the fact that no meaningful correlation was found between the attention deficit score and trauma intensity. The results also showed that trauma severity in adolescents with ADHD and without ADHD was different and it was greater in the ADHD group than the control group. This is in line with the results of Biederman et al (31). We could observe a statistically negative correlation between the components of ADHD and oppositional/impulsive and the weak economic and social situation. In this regard, in the weak economic and social situation, the above components were of great value. This finding is inconsistent with the study conducted by Ziaoddini and Shafizadeh (32). Also, no meaningful correlation between the frequency of conduct disorder and hyperactivity with socio-economic status, was reported. This is consistent with the study by Kouchakzadeh et al (30) indicating that there was a statistically meaningful correlation between variables such as education level of parents and family income with inattention disorder and hyperactivity. It is likely that cognitive and emotional development of children in low-income families will be impaired. In poor families, mother and children are more exposed to stressful events, caring problems due to mother's occupation, and lack of physical and mental health. In this regard, Hudson and Rapee in a research came to this conclusion that most social abnormalities were seen in the slum of Chicago, and the highest rate of unemployed people was from discrete families and criminals (33). In the studies conducted by Brault and Lacourse (34) and Ghanizadeh (35), ADHD prevalence was high in families with lower socio-economical conditions. The research findings by Duncan et al (36) showed that there was a meaningful correlation between the indicators of socio-economical condition, mental and physical health, and the prevalence of diseases with low 
social prestige, low income and low level of education. In addition, the failure in different stages of treatment was seen in patients with low socio-economical condition. Also, examining the indicators between socio-economical condition and physical disease, they found that welfare and family income were among the indicators of fixed socio-economical condition, and were more significant in women than men and in adolescents than the elderly. Analyzing the risk factors of diseases and the physical injuries such as trauma indicates that the education level was meaningfully correlated with risk factors for physical injuries, and having a healthy lifestyle but in those with a lower education than a high school diploma is more unlikely.

Taking into account the influence of demographic characteristics of parents on the mental disorders of adolescents in a simple way without conducting further accurate studies is impossible.

Acquiring a different result is possible by using other research methodologies with the aim of determining the exact effect of factors, such as parental age, maternal employment and socio-economical class and using other research tools, such as interviews or observations.

The different method applied in this study may be the reason for inconsistent findings of our research. Some studies have indicated that people with ADHD are more interested in participating in high-risk behaviors than their peers (37). In addition, some studies have indicated that this disorder increases the risk of injuries and pedestrian accidents in adolescents. The weak attentive skills and high impulsivity for crossing the street could increase the damage risk (14). Fischer et al (38) studied the behaviors related to driving and its consequences during the adolescence and childhood of the hyperactive children. The results showed that in traffic accidents there was a significant difference between these people with the control group. But, the study by Amiri et al (21) comparing 70 patients with musculoskeletal trauma caused by driving accidents with 70 people as a control group with an experience of repeated trauma indicated that there was no relationship between ADHD in the adulthood and the trauma caused by driving accidents.

\section{Conclusion}

The results showed that there was a statistically positive relationship between the severity of trauma and ADHD score, hyperactivity, and oppositional/impulsivity. But there was no statistically meaningful relationship between attention deficit score and trauma severity. Also, the results indicated that trauma severity in adolescents with ADHD and without ADHD was different. The severity was bigger in $\mathrm{ADHD}$ group in comparison to normal individuals. In addition, there was a negative significant correlation between components of ADHD and oppositional// impulsivity with socio-economic conditions.

Given the deep influences of trauma on the human and financial resources and the high rate of accidents in children, it is necessary for policymakers to plan consistent programs for screening, education, and prevention. Also, we should take the precaution of helping such children to prevent forthcoming problems.

\section{Limitations of study}

In this study we did not consider other traumas resulted from burning, committing suicide, or self-destruction and child abuse. We recommend conducting other studies in adults groups and considering other trauma cases. We also suggest studies to investigate the relationship between trauma severity and attention deficit/hyperactivity among both sexes.

\section{Acknowledgements}

We express our thanks to all patients and parents who participated in this study.

\section{Ethical issues}

This study was approved by Regional Ethical Committee with number TBZMED.REC.1395.263.

\section{Authors' contributions}

All authors confirm that they have participated in study design, data gathering, analysis and preparing the manuscript based on recommendations of the International Committee of Medical Journal Editors.

\section{References}

1. American Psychiatric Association (APA). Diagnostic and statistical manual of mental disorders: DSM-IV-TR ${ }^{\oplus}$. 5th ed. Washington (DC): APA Publishing; 2000.

2. Biederman J, Faraone SV. Attention-deficit hyperactivity disorder. Lancet 2005; 366(9481): 237-48. doi: 10.1016/ S0140-6736(05)66915-2.

3. Tannock R. Rethinking ADHD and LD in DSM-5: proposed changes in diagnostic criteria. J Learn Disabil 2013; 46(1): 5-25. doi: 10.1177/0022219412464341.

4. Frick P, Moffitt T. A proposal to the DSM-V childhood disorders and the ADHD and disruptive behavior disorders work groups to include a specifier to the diagnosis of conduct disorder based on the presence of callousunemotional traits. Washington, DC: American Psychiatric Association; 2010.

5. Froehlich TE, Lanphear BP, Epstein JN, Barbaresi WJ, Katusic SK, Kahn RS. Prevalence, recognition, and treatment of attention deficit/hyperactivity disorder in a national sample of USchildren. Arch Pediatr Adolesc Med 2007; 161(9): 857-64. doi: 10.1001/archpedi.161.9.857.

6. Riley AW, Spiel G, Coghill D, Döpfner M, Falissard B, Lorenzo MJ, et al. Factors related to health-related quality of life (HRQoL) among children with ADHD in Europe at entry into treatment. Eur Child Adolesc Psychiatry 2006; 15 Suppl 1: I38-45. doi: 10.1007/s00787-006-1006-9.

7. Harpin VA. The effect of ADHD on the life of an individual, their family, and community from preschool to adult life. Arch Dis Child 2005; 90 Suppl 1: i2-7. doi: 10.1136/ adc.2004.059006.

8. Davids E, Gastpar M. Attention deficit hyperactivity disorder and borderline personality disorder. Prog Neuropsychopharmacol Biol Psychiatry 2005; 29(6): 865- 
77. doi: 10.1016/j.pnpbp.2005.04.033.

9. Wender PH. Attention-deficit hyperactivity disorder in adults. Psychiatr Clin North Am 1998; 21(4): 761-74. doi: 10.1016/S0193-953X(05)70039-3.

10. Faraone SV, Biederman J, Spencer T, Wilens T, Seidman LJ, Mick E, et al. Attention-deficit/hyperactivity disorder in adults: an overview. Biol Psychiatry 2000; 48(1): 9-20.

11. Bennett DS, Sullivan MW, Lewis M. Neglected children, shame-proneness, and depressive symptoms. Child Maltreat 2010; 15(4): 305-14. doi: 10.1177/10775559510379634.

12. Farmer JE, Peterson L. Injury risk factors in children with attention deficit hyperactivity disorder. Health Psychol 1995; 14(4): 325-32.

13. DiScala C, Lescohier I, Barthel M, Li G. Injuries to children with attention deficit hyperactivity disorder. Pediatrics 1998; 102(6): 1415-21.

14. Pendleton S, Stavrinos D, Cotney R, Bridgmon K, Schwebel DC. How temperament and ADHD predict child pedestrian injury. Proceedings of the 2009 Society for the Advancement of Violence and Injury Research Conference; 2009 Mar 5-6; Atlanta, GA, USA.2009

15. Siesjö BK. Basic mechanisms of traumatic brain damage. Ann Emerg Med 1993; 22(6): 959-69.

16. Yousefzadeh S, Dafchahi MA, Maleksari HM, Moghadam $\mathrm{AD}$, Hemati H, Shabani S. Epidemiology of injuries and their causes among traumatic patients admitted into Poursina hospital, Rasht. Journal of Kermanshah University of Medical Sciences 2007;11(3): 286-95. [In Persian].

17. Schwebel DC, Roth DL, Elliott MN, Visser SN, Toomey SL, Shipp EM, et al. Association of externalizing behavior disorder symptoms and injury among fifth graders. Acad Pediatr 2011;11(5): 427-31. doi: 10.1016/j.acap.2011.03.003.

18. Lara C, Fayyad J, De Graaf R, Kessler RC, Aguilar-Gaxiola $S$, Angermeyer $M$, et al. Childhood predictors of adult attention-deficit/hyperactivity disorder: results from the World Health Organization World Mental Health Survey Initiative. Biol Psychiatry 2009; 65(1): 46-54. doi: 10.1016/j. biopsych.2008.10.005.

19. Zargarinezhad G, Yekeh Y. Efficacy of parent's training on problem behaviors in ADHD children. Psychological Studies 2007; 3(2): 29-48. [In Persian].

20. Kaufmann CR, Maier RV, Rivara FP, Carrico CJ. Evaluation of the pediatric trauma score. JAMA 1990; 263(1): 69-72. doi:10.1001/jama.1990.03440010067032.

21. Amiri S, Shafiee-Kandjani AR, Fakhari A, Abdi S, Golmirzaei J, Akbari Z. Psychiatric comorbidities in ADHD children: an iranian study among primary school students. Arch Iran Med 2013; 16(9): 513-7.

22. Abolhassanzadeh M, Shafiee-Kandjani AR, Vaziri Z, Molavi P, Sadeghi-Movahhed F, Noorazar G, et al. The prevalence and risk factors of attention deficit hyperactivity disorder among the elementary school students in Ardabil, Iran, in 2011-2012. J Anal Res Clin Med 2016; 4(3): 146-52. doi:10.15171/jarcm.2016.024.

23. Bener A, Qahtani RA, Abdelaal I. The prevalence of ADHD among primary school children in an Arabian society. J Atten Disord 2006; 10(1): 77-82. doi: 10.1177/1087054705284500.

24. Mugnaini D, Masi G, Brovedani P, Chelazzi C, Matas M, Romagnoli C, et al. Teacher reports of ADHD symptoms in Italian children at the end of first grade. Eur Psychiatry 2006; 21(6): 419-26. doi:10.1016/j.eurpsy.2005.04.011.

25. Rappley MD. Clinical practice. Attention deficithyperactivity disorder. N Engl J Med 2005; 352(2): 165-73. doi: 10.1056/NEJMcp032387.

26. Shams-Esfandabadi H, Emami Pour S, Sadrossadat G. A study on prevalence of behavioral disorders in primary school students in Abhar. Archives of Rehabilitation 2003; 4(1): 34-41. [In Persian].

27. Alishahi M, Deh Bozorgi GR, Dehghan B. Prevalence rate of attention deficit hyperactivity disorder among the students of primary schools. Zahedan J Rese Med Sci 2003; 5 (1): 61-7. [In Persian].

28. Shahim S, Mehrangiz L, Yousefi F. Prevalence of attention deficit hyperactivity disorder in a group of elementary school children. Iranian Journal of Pediatrics 2007; 17 (Suppl 2): 211-6. [In Persian].

29. Kaya A, Taner Y, Guclu B, Taner E, Kaya Y, Bahcivan H, et al. Trauma and adult attention deficit hyperactivity disorder. J Int Med Res 2008; 36(1): 9-16. doi: $10.1177 / 147323000803600102$.

30. Kouchakzadeh Talami S, Namazi A, Zarkesh M. Symptoms related to attention deficit hyperactivity disorder among preschool children based on parents report. J Holist Nurs Midwifery 2015; 25(1): 35-44. [In Persian].

31. Biederman J, Petty CR, Spencer TJ, Woodworth KY, Bhide $\mathrm{P}, \mathrm{Zhu} \mathrm{J}$, et al. Examining the nature of the comorbidity between pediatric attention deficit/hyperactivity disorder and post-traumatic stress disorder. Acta Psychiatr Scand 2013; 128(1): 78-87. doi: 10.1111/acps.12011.

32. Ziaoddini H, Shafizadeh N. The Epidemiology of Attention Deficit Hyperactivity Disorder and Conduct Disorder in Elementary School children in the City of Sirjan. Iranian Journal of Psychiatry and Clinical Psychology 2006; 11(4): 419-25. [In Persian].

33. Hudson JL, Rapee RM. Parent-child interactions and anxiety disorders: an observational study. Behav Res Ther 2001; 39(12): 1411-27. doi: 10.1016/S0005-7967(00)001078.

34. Brault MC, Lacourse É. Prevalence of prescribed attentiondeficit hyperactivity disorder medications and diagnosis among Canadian preschoolers and school-age children: 1994-2007. Can J Psychiatry 2012; 57(2): 93-101. doi: $10.1177 / 070674371205700206$

35. Ghanizadeh A. Distribution of symptoms of attention deficit-hyperactivity disorder in schoolchildren of Shiraz, south of Iran. Arch Iran Med 2008; 11(6): 618-24.

36. Duncan GJ, Daly MC, McDonough P, Williams DR. Optimal indicators of socioeconomic status for health research. Am J Public Health 2002; 92(7): 1151-7.

37. Nichols JQVA. Human factors and driving behaviors differentiating high risk and low risk drivers with attention deficit hyperactivity disorder [dissertation]. Charlottesville, VA: University of Virginia; 2012.

38. Fischer M, Barkley RA, Smallish L, Fletcher K. Hyperactive children as young adults: driving abilities, safe driving behavior, and adverse driving outcomes. Accid Anal Prev 2007; 39(1): 94-105. doi: 10.1016/j.aap.2006.06.008. 